\title{
LAPLACE TRANSFORMS FOR SUPEREXPONENTIAL FUNCTIONS
}

\author{
MICHAEL A. B. DEAKIN ${ }^{1}$
}

(Received 4 March 1992; revised 18 September 1992)

\begin{abstract}
It is usually stated that the Laplace transform cannot be applied to most superexponential functions; indeed this limitation is sometimes represented as a deficiency of the technique. It is shown here that a generalisation serves to overcome much of the force of this objection. The generalisation is based on one first proposed over fifty years ago, but which is not widely known, nor was it ever worked out in detail.
\end{abstract}

\section{Introduction}

The Laplace transform is normally defined by

$$
f(s)=\int_{0}^{\infty} e^{-s t} F(t) d t,
$$

and this definition, naturally, fails to apply if the defining integral diverges. This has been seen as a limitation by (for example) Liverman [8], Krabbe [7] and most recently Výborný [14], all of whom adduce simple differential or integral equations to which the transform, so defined, cannot be applied. Krabbe [7, page 103] does remark of two examples that "if this fact is ignored, the answers obtained will turn out to be correct", but the examples in question are relatively simple and although the $F(t)$ involved are superexponential, he is nowhere under the necessity of exhibiting explicitly the corresponding $f(s)$.

The deficiency of the usual definition (1.1) is encountered quite early in that it precludes the use of the Laplace transform for a straightforward investigation of the relatively simple differential equation:

$$
\left(a_{2} t+b_{2}\right) X^{\prime \prime}(t)+\left(a_{1} t+b_{1}\right) X^{\prime}(t)+\left(a_{0} t+b_{0}\right) X(t)=0 .
$$

\footnotetext{
${ }^{1}$ Department of Mathematics, Monash University, Clayton, Victoria 3168, Australia

(C) Australian Mathematical Society, 1994, Serial-fee code 0334-2700/94
} 
This equation has a number of standard forms (see, for example [4, page 249]) of which

$$
X^{\prime \prime}(t)-t X^{\prime}(t)+a X(t)=0
$$

is not amenable to a straightforward Laplace treatment as at least one (and often both) of its solutions leads to a divergent Laplace integral. (The same remarks apply to another standard form

$$
X^{\prime \prime}(t)-t X^{\prime}(t)=0
$$

but in this case the matter is easily rectified.)

\section{Asymptotic Laplace integrals}

The concept of an asymptotic Laplace integral was introduced by Vignaux [14] in a paper that appeared in 1939 but which has gone almost completely unnoticed. Vignaux defined

$$
f(s) \sim \int_{0}^{\infty} e^{-s t} F(t) d t
$$

if

$$
f(s)=\int_{0}^{T} e^{-s t} F(t) d t+o\left(s^{-s T}\right)
$$

for all $T>0$.

The following properties of asymptotic Laplace integrals are readily proved. First: if the integral on the right of (2.1) converges then the value so attained satisfies (2.1). Secondly, (2.1) is linear. Thirdly, if (2.1) holds and also

$$
f^{\prime}(s) \sim \int_{0}^{\infty} e^{-s t} G(t) d t
$$

then

$$
G(t)=-t F(t)
$$

Vignaux also showed that (2.1) implies

$$
\int_{s_{0}}^{s} f(u) d u-\ln \left(s / s_{0}\right) \sim \int_{0}^{\infty} e^{-s t}\left\{\frac{F(0)-F(t)}{t}\right\} d t+C,
$$

where $C$ is a constant whose value depends on $s_{0}$.

There is also a convolution theorem: if (2.1) holds and an analogous relation connects $g(s), G(t)$, then

$$
f(s) g(s) \sim \int_{0}^{\infty} e^{-s t} \int_{0}^{t} F(u) G(t-u) d u d t .
$$


Relation (2.1) does not define $f(s)$ uniquely. Berg [2, Sections 50-51] offers one suggestion as a means of overcoming this problem, but it gives a clumsy expression for $\mathscr{L}\left\{\exp t^{2}\right\}$ and he notes that a different possibility gives a simpler result, although neither of these does he exhibit explicitly.

In fact neither Vignaux nor Berg gives any explicitly evaluated transforms nor makes any application of the results derived for the asymptotic integrals.

\section{Asymptotic Series}

Suppose $F(t)$ to be analytic for all $t \geq t_{0}$ (say) and let $T>t_{0}$. Set up the expression

$$
\phi\{s ; T \mid F(t)\}=\frac{1}{s} e^{-s t}\left\{F(T)+\frac{1}{s} F^{\prime}(T)+\frac{1}{s^{2}} F^{\prime \prime}(T)+\ldots\right\} .
$$

This formal expansion possesses the following properties.

$$
\phi\{s ; T \mid a F(t)+b G(t)\}=a \phi\{s ; T \mid F(t)\}+b \phi\{s ; T \mid G(t)\},
$$

where $a, b$ are constants.

$$
\begin{aligned}
\phi\left\{s ; T \mid e^{a t} F(t)\right\} & =\phi\{s-a ; T \mid F(t)\}, \\
\phi\{s ; T \mid F(t-a)\} & =e^{-a s} \phi\{s ; T-a \mid F(t)\}, \\
\phi\{s ; T \mid F(a t)\} & =\frac{1}{a} \phi\{s / a ; a T \mid F(t)\},
\end{aligned}
$$

where $a$ is a constant in each case and $a T, T-a$ are both greater than $t_{0}$.

$$
\begin{aligned}
\phi\left\{s ; T \mid F^{(n)}(t)\right\} & =s^{n} \phi\{s ; T \mid F(t)\}-e^{-s T} \sum_{k=0}^{n-1} s^{k} F^{(n-k-1)}(T), \\
\phi\left\{s ; T \mid \int_{0}^{t} F(u) d u\right\} & =\frac{1}{s} \phi\{s ; T \mid F(t)\}-\frac{1}{s} e^{-s T} \int_{0}^{T} F(u) d u, \\
\phi\left\{s ; T \mid t^{n} F(t)\right\} & =(-d / d s)^{n} \phi\{s ; T \mid F(t)\},
\end{aligned}
$$

where the differentiation is applied term by term, and

$$
\phi\{s ; T \mid F(t) / t\}=\int_{s}^{\infty} \phi\{s ; T \mid F(t)\} d u
$$

where the integration is applied term by term.

The proofs of all these statements are trivial and may be supplied by the reader, who will note the connection between $(3.2-3.10)$ and the corresponding properties of $\mathscr{L}\{F(t)\}$. 
We also note two further properties,

$$
\frac{\partial}{\partial T} \phi\{s ; T \mid F(t)\}=-e^{-s T} F(T)
$$

and its corollary

$$
\phi\left\{s ; T_{1} \mid F(t)\right\}-\phi\left\{s ; T_{2} \mid F(t)\right\}=\int_{T_{1}}^{T_{2}} e^{-s T} F(T) d T .
$$

Especially in the case where $F(t)$ is analytic in the neighbourhood of 0 and $T$ may be taken to be zero, we may interpret $\phi\{s ; T\{F(t)\}$ as an asymptotic series in $1 / s$.

Since, in all cases, $F(t)$ is analytic at $t=T$, the Taylor expansion of $F(t)$ about that point is convergent, so that $F^{(n)}(T)=O\left((n !)^{2}\right)$, and hence $F^{(n)}(T)=O((2 n)$ !). If we sum the asymptotic series by Ritt's method [10, page 23-24] we obtain a bound on the remainder that enables us to apply a classic theorem due to Watson [15, Theorem IV] and so obtain a unique analytic sum in a region $R(s) \geq \beta$ (say). Olver [10, page 30] has remarked of such results that although they "are of great theoretical interest, practical applications are rare". One reason for this is the difficulty experienced in relating the analytic functions constructed by Ritt's technique to the functions discussed and tabulated in standard reference texts. Accordingly, although the possibility of a unique determination of $\phi\{s ; T \mid F(t)\}$ is noted, no further use will be made of it.

\section{Finite Laplace transforms}

The finite Laplace transform

$$
f_{T}(s)=\int_{0}^{T} e^{-s t} F(t) d t=\mathscr{L}_{T}\{F(t)\}
$$

has been investigated before $[2,9]$ but mainly with the understanding that $T$ is fixed. One may however allow a variable $T$, in which case the following results are readily proved.

$$
\begin{aligned}
\mathscr{L}_{T}\left\{F(t) e^{a t}\right\} & =f_{T}(s-a), \\
\mathscr{L}_{T}\{F(t-a) \mathscr{U}(t-a)\} & =f_{T-a}(s) e^{-a s} \quad(T>a),
\end{aligned}
$$

where $\mathscr{U}$ denotes the unit step function,

$$
\begin{aligned}
& \mathscr{L}_{T}\{F(a t)\}=\frac{1}{a} f_{a T}(s / a), \\
& \mathscr{L}_{T}\left\{F^{(n)}(t)\right\}=s^{n} f_{T}(s)-\sum_{k=0}^{n-1} s^{k}\left[F^{(n-k-1)}(0)-e^{-s T} F^{(n-k-1)}(T)\right],
\end{aligned}
$$




$$
\begin{aligned}
\mathscr{L}_{T}\left\{\int_{0}^{t} F(u) d u\right\} & =\frac{1}{s} f_{T}(s)-\frac{1}{s} e^{-s T} \int_{0}^{T} F(u) d u, \\
\mathscr{L}_{T}\left\{t^{n} F(t)\right\} & =(-d / d s)^{n} f_{T}(s), \\
\mathscr{L}_{T}\left\{\frac{F(t)}{t}\right\} & =\int_{s}^{\infty} f_{T}(u) d u, \\
\lim _{s \rightarrow \infty} f_{T}(s) & =0 \\
\lim _{t \rightarrow 0} F(t) & =\lim _{s \rightarrow \infty} s f_{T}(s), \\
\lim _{s \rightarrow \infty} s f_{T}(s) & =F(0) .
\end{aligned}
$$

$$
\text { If } F(t) \sim G(t) \text { as } t \rightarrow 0 \text {, then } f_{T}(s) \sim g_{T}(s) \text { as } s \rightarrow \infty \text {. }
$$

We may note that $f_{T}(s)$ is analytic in the whole $s$-plane.

\section{The generalised Laplace transform}

Define the generalised Laplace transform of $F(t)$ by

$$
f(s)=\mathscr{L}_{G}\{F(t)\}=\mathscr{L}_{T}\{F(t)\}+\phi\{s: T \mid F(t)\},
$$

where $F(t)$ is assumed integrable on $\left[0, t_{0}\right]$ and analytic on $\left[t_{0}, \infty\right]$. We take $T>t_{0}$. Then the expression $f(s)$ is readily seen to be independent of $T$ in consequence of (3.10) and (3.11). Furthermore, if $F(t)$ possesses a Laplace transform in the usual sense then $f(s)$ will represent the standard Laplace transform. In this case

$$
\lim _{s \rightarrow \infty} \phi\{s ; T \mid F(t)\}=0
$$

$f(s)$ defined by (5.1) shares many properties of the usual Laplace transform. $\mathscr{L}_{G}$ is a linear functional. Moreover,

$$
\mathscr{L}_{G}\left\{e^{a t} F(t)\right\}=f(s-a) .
$$

The other shift theorem also holds:

$$
\mathscr{L}_{G}\{F(t-a) \mathscr{U}(t-a)\}=e^{-a s} f(s)
$$

(This result is the one that necessitates the apparently cumbersome definition (5.1) in place of the simpler equation achieved by setting $T=0$ there.) Other standard results also carry over.

$$
\mathscr{L}_{G}\{F(a t)\}=\frac{1}{a} f(s / a),
$$




$$
\begin{aligned}
\mathscr{L}_{G}\left\{F^{(n)}(t)\right\} & =s^{n} f(s)-\sum_{k=0}^{n-1} s^{k} F^{(n-k-1)}(0), \\
\mathscr{L}_{G}\left\{\int_{0}^{t} F(u) d u\right\} & =\frac{1}{s} f(s), \\
\mathscr{L}_{G}\left\{t^{n} F(t)\right\} & =(-d / d s)^{n} f(s), \\
\mathscr{L}_{G}\left\{\frac{F(t)}{t}\right\} & =\int_{s}^{\infty} f(u) d u .
\end{aligned}
$$

The $F\left(t^{2}\right)$ formula

$$
\mathscr{L}_{G}\left\{F\left(t^{2}\right)\right\}=\frac{1}{2 \sqrt{\pi}} \int_{0}^{\infty} u^{-1 / 2} \exp \left(-\frac{s^{2}}{4 u}\right)\left[f_{T}(u)+\phi\{u ; T \mid F(t)\}\right] d u
$$

also obtains and may readily be proved by showing that

$$
\mathscr{L}_{G}\left\{F\left(t^{2}\right)\right\}=\frac{1}{2 \sqrt{\pi}} \int_{0}^{\infty} u^{-1 / 2} \exp \left(-\frac{s^{2}}{4 u}\right) f_{T}(u) d u
$$

and

$$
\phi\left\{s ; T \mid F\left(t^{2}\right)\right\}=\frac{1}{2 \sqrt{\pi}} \int_{0}^{\infty} u^{-1 / 2} \exp \left(-\frac{s^{2}}{4 u}\right) \phi\{u ; T \mid F(u)\} d u .
$$

\section{Explicit transform pairs}

In the case of several well-known superexponential functions, the asymptotic series arising as $\phi\{s ; T \mid F(t)\}$ are standard representations of explicitly given functions. A number of these are listed here. In each case it is sufficient to take $T=0$.

$$
\mathscr{L}_{G}\left\{\exp t^{2}\right\} \sim \operatorname{daw}(s / 2)
$$

where daw $(s)$ is Dawson's integral [12, pages 405-410]. This follows immediately from the asymptotic expansion

$$
\mathscr{L}_{G}\left\{\exp \left(t^{3} / 3\right)\right\} \sim-\pi \mathrm{Hi}(s),
$$

where $\mathrm{Hi}(s)$ is the second related Airy function. This is most conveniently derived from the expansion given in [11]

$$
\mathscr{L}_{G}\left\{\exp \left(t^{2}\right) \operatorname{erf} t\right\} \sim \frac{1}{2 \sqrt{\pi}} \exp \left(-\frac{s^{2}}{4}\right) \operatorname{Ei}\left(\frac{s^{2}}{4}\right),
$$


where erf represents the error function and Ei the exponential integral in the notation of [1]. One of several routes to this is the application of (5.5) to the known result

$$
\mathscr{L}\{\text { daw } t\}=-\frac{1}{4} \exp \left(\frac{s^{2}}{4}\right) \operatorname{Ei}\left(\frac{s^{2}}{4}\right) .
$$

If $I_{0}(t)$ is the modified Bessel function of the first kind,

$$
\mathscr{L}_{G}\left\{I_{0}\left(t^{2}\right)\right\} \sim \frac{\sqrt{\pi}}{2} \Phi_{1}\left(\frac{1}{2}, \frac{1}{2}, 1 ;-1,-\frac{s^{2}}{4}\right),
$$

where $\Phi_{1}$ is a hypergeometric function of two variables [3, page 139]. The result may be discovered by application of (5.10).

Set

$$
X(t)=\operatorname{Ei}\left(t^{2}\right)-2 \ln t
$$

and hence find $X^{\prime}(t)$. Taking a generalised Laplace transform, and noting that $X(0)=$ $\gamma$, find

$$
\mathscr{L}_{G}\left\{\operatorname{Ei}\left(t^{2}\right)\right\} \sim \frac{\gamma}{s}-\frac{2 \ln s}{s}+\frac{2}{s} \int_{0}^{s}\left[\operatorname{daw}\left(\frac{u}{2}\right)-\frac{1}{u}\right] d u .
$$

There are several routes to $\mathscr{L}_{G}\{\mathrm{Bi}(t)\}$ where $\mathrm{Bi}(t)$ is the second Airy function. We find, in terms of incomplete gamma functions [1],

$$
\mathscr{L}_{G}\{\mathrm{Bi}(t)\} \sim \frac{1}{\sqrt{3}} \exp \left(-\frac{s^{3}}{3}\right)\left\{\frac{1}{\Gamma\left(\frac{2}{3}\right)} \gamma\left(\frac{2}{3},-\frac{s^{3}}{3}\right)-\frac{1}{\Gamma\left(\frac{1}{3}\right)} \gamma\left(\frac{1}{3},-\frac{s^{3}}{3}\right)\right\} .
$$

[Note that many published values for the classical Laplace transforms of $\operatorname{Ai}( \pm t)$, $\mathrm{Bi}(-t)$ are either misprinted or use non-standard notations. A list of relevant literature is given in [6] but this does not explicitly correct all the various mistakes in previous papers.]

$$
\mathscr{L}_{G}\left\{\exp \left(e^{t}-1\right)\right\} \sim \frac{(-1)^{s}}{e} \Gamma(-s,-1),
$$

where $\Gamma(s, a)$ is an incomplete gamma function [1]. This interesting result will be the subject of a later paper. A formal Laplace transform of $\exp \left(e^{t}-1\right)$ has previously been used in a combinatorial context by Flajolet and Schott [5].

Applying (5.10) to the Laplace transform of $e^{t}$ and using (6.1) gives

$$
\mathscr{L}_{G}\left\{\frac{1}{\sqrt{t}(1-t)}\right\} \sim 2 \sqrt{\pi} \operatorname{daw}(\sqrt{s}) .
$$

In fact one may prove that

$$
\mathscr{L}\left\{\frac{1}{\sqrt{t}(1-t)}\right\}=2 \sqrt{\pi} \operatorname{daw}(\sqrt{s})
$$


if the defining integral for the left-hand expression is interpreted as a Cauchy principal value. This may be proved with relative ease by a number of traditional methods. It may be a new result, for I have been unable to find it in the literature. See however a related result given by Erdélyi [4, page 136].

Finally we may note that $(6.1),(6.3)$ are special cases of more general results. The solutions of (1.3) are written in several standard forms of which is it most convenient to use $\exp \left[t^{2} / 4\right] E_{a}^{(0)}(t)$ and $\exp \left[t^{2} / 4\right] E_{a}^{(1)}(t)$, where $E_{a}^{(0)}(t)$ and $E_{a}^{(1)}(t)$ are Weber's parabolic cylinder functions [1, page 509]. Applying a generalised Laplace transform to (1.3) results in the relations:

$$
\begin{aligned}
& \mathscr{L}_{G}\left\{\exp \left(\frac{t^{2}}{4}\right) E_{a}^{(0)}(t)\right\} \sim \sqrt{2} s^{-(1+a)} \exp \left(-\frac{s^{2}}{2}\right) \int_{s_{0}}^{s} u^{a} \exp \left(\frac{u^{2}}{2}\right) d u, \\
& \mathscr{L}_{G}\left\{\exp \left(\frac{t^{2}}{4}\right) E_{a}^{(1)}(t)\right\} \sim 2 s^{-(1+a)} \exp \left(-\frac{s^{2}}{2}\right) \int_{s_{0}}^{s} u^{a} \exp \left(\frac{u^{2}}{2}\right) d u,
\end{aligned}
$$

where $s_{0}$ is arbitrary, and may be chosen for simplicity in the evaluated integral.

If $a$ is zero or positive integral, (1.3) is a form of the Hermite equation, and one or another of its solutions is polynomial, the second being superexponential. For other values of $a$, both solutions are superexponential. Formulae (6.1), (6.3) derive from the case $a=-1$.

Thus if all cases of (1.2) are to be solved by means of the Laplace transform this can be done using the generalised form and cannot be done without it.

\section{The inverse transform}

The standard formula for the inverse transform is

$$
\mathscr{L}^{-1}\{f(s)\}=\frac{1}{2 \pi i} \int_{C} e^{-s t} f(s) d s,
$$

where $C$ is usually taken to be a vertical line at $x=\gamma$ for suitably large $\gamma$. It may also be taken to be a circle centred on $\infty$, provided $\infty$ is an isolated singularity.

Put $s=1 / u$ in (7.1) to find

$$
\mathscr{L}^{-1}\{f(s)\}=\frac{1}{2 \pi i} \int_{-C^{\prime}} e^{t / u} f\left(\frac{1}{u}\right) u^{-2} d u,
$$

where $-C^{\prime}$ is a circle centred on the origin, containing no other singularities of the integrand and traversed counter-clockwise. If now $t>T$,

$$
F(t)=\sum_{n=0}^{\infty} \frac{1}{n !} F^{(n)}(T)(t-T)^{n} .
$$


Thus

$$
\phi\{s ; T \mid F(t)\}=s^{-1} e^{-s T} \sum_{n=0}^{\infty} s^{-n} f^{(n)}(T) .
$$

If we insert this into the integrand of (7.2), the coefficient of $1 / u$ is found to be the right-hand side of (7.3).

If $t<T$, the original function $F(t)$ is generated from $L_{T}\{F(t)\}$ by standard theory. This is not needed in the case $T=0$. It is a simple exercise to apply (7.2) to daw $(s / 2)$ to find

$$
\mathscr{L}^{-1}\{\operatorname{daw}(s / 2)\}=\exp \left(t^{2}\right)
$$

\section{Acknowledgements}

I thank Dr P. Flajolet for his support, encouragement and illuminating discussion, and Dr A. A. Inayat-Hussain who directed me to the literature on the Laplace transforms of Airy function. Parts of this work were undertaken during sojourns at Universitas Sebelas Maret (Indonesia) and the University of Wisconsin at Madison. I thank those institutions for their hospitality.

\section{References}

[1] M. Abramowitz and I. A. Stegun (eds.), Handbook of mathematical functions (Dover Publications, New York, 1968).

[2] L. Berg, Introduction to the operational calculus (North Holland Publishing Co., Amsterdam, 1967).

[3] A. Erdélyi (ed.), Higher trancendental functions, Volume I (McGraw Hill Book Co., New York, 1953).

[4] A. Erdélyi (ed.), Tables of integral tranforms, Volume I (McGraw Hill Book Co., New York, 1954).

[5] P. Flajolet and R. Schott, "Non-overlapping partitions, continued fractions, Bessel functions and a divergent series", Europ. J. Combinatorics 11 (1990) 421-432.

[6] A. A. Inayat-Hussein and M. J. Buckingham, "On the Laplace transform of the $G$ function", $J$. Phys. A: Math. Gen. 19 (1986) L875-L876.

[7] G. Krabbe, Operational calculus (Springer-Verlag, Berlin, 1970).

[8] T. P. G. Liverman, Generalized functions and direct operational methods (Prentice-Hall, Englewood Cliffs, N. J., 1964).

[9] J. Mikusiński, "Le calcul opérationnel d'intervalle fini”, Studia Mathematica 15 (1956) 225-251.

[10] F. W. J. Oliver, Asymptotics and special functions (Academioc Press, New York, 1974).

[11] M. Rothman, "Tables of the integrals and differential coefficients of $\mathrm{Gi}(+x)$ and $\mathrm{Hi}(-x)$ ", Quart. J. Mech. Appl. Math. (1954) 379-384.

[12] J. Spanier and K. B. Oldham, An atlas of functions (Hemisphere Publishing Corp., Washington, 1987).

[13] J. C. Vignaux, "Sugli integrali di Laplace asintotici", Atti Accad. naz. Lincei, Rend. Cl. Sci. fis. mat. 29 (6 ser.) (1939) 396-402.

[14] R. Výborný, "Operational calculus, Laplace transforms or an algebraic approach", Aust. Math. Soc. Gaz. 18 (1991) 71-78.

[15] G. N. Watson, “A theory of asymptotic series”, Proc. Roy. Soc. A11 (1911) 279-313. 